\title{
Development of a Novel Intraoperative Neuromonitoring System Using a Surface Pressure Sensor to Detect Muscle Movement: A Rabbit Model Study
}

\author{
Eui-Suk Sung ${ }^{1}$ (D) $\cdot$ Jin-Choon Lee ${ }^{1} \cdot$ Sung-Chan Shin ${ }^{2} \cdot$ Hyun-Geun Kwon ${ }^{2} \cdot$ Min-Sik Kim ${ }^{1}$ Dong-Jo Kim ${ }^{1}$ \\ Jung-Hoon Ro ${ }^{3} \cdot$ Byung-Joo Lee $^{2}$ (i) \\ ${ }^{I}$ Department of Otolaryngology, Head and Neck Surgery and Research Institute for Convergence of Biomedical Science and Technology, \\ Pusan National University College of Medicine, Yangsan; \\ ${ }^{2}$ Department of Otolaryngology, Head and Neck Surgery and Medical Research Institute, Pusan National University College of Medicine, Busan; \\ ${ }^{3}$ Department of Biomedical Engineering, Pusan National University Hospital, Busan, Korea
}

Objectives. False-negative or false-positive responses in intraoperative neuromonitoring (IONM) using electromyography (EMG) in thyroid surgery pose a challenge. Therefore, we developed a novel IONM system that uses a surface pressure sensor instead of EMG to detect muscle twitching. This study aimed to investigate the feasibility and safety of a new IONM system using a piezo-electric surface pressure sensor in an experimental animal model.

Methods. We developed the surface pressure sensor by modifying a commercial piezo-electric sensor. We evaluated the stimulus thresholds to detect muscle movement, as well as the amplitude and latency of the EMG and surface pressure sensor in six sciatic nerves of three rabbits, according to the stimulus intensity.

Results. The surface pressure sensor detected the muscle movements in response to a $0.1 \mathrm{~mA}$ stimulation of all six sciatic nerves. There were no differences in the thresholds of stimulus intensity between the surface pressure sensor and EMG recordings to detect muscle movements.

Conclusion. It is possible to measure the change in surface pressure by using a piezo-electric surface pressure sensor instead of EMG to detect muscle movement induced by nerve stimulation. The application of IONM using a piezo-electric surface pressure sensor during surgery is noninvasive, safe, and feasible. Measuring muscle twitching to identify the state of the nerves using the novel IONM system can be an alternative to recording of EMG responses.

Keywords. Intraoperative Neurophysiological Monitoring; Surface Pressure Sensor; Electromyography; Recurrent Laryngeal Nerve; Thyroidectomy

\section{INTRODUCTION}

The prevention of motor nerve injury during surgery is crucial. However, nerve injuries inevitably occur because of an inade-

\footnotetext{
- Received August 18, 2018

Revised September 25, 2018

Accepted October 18, 2018

- Corresponding author: Byung-Joo Lee

Department of Otolaryngology, Head and Neck Surgery, Medical Research Institute and Pusan National University College of Medicine,

305 Gudeok-ro, Seo-gu, Busan 49241, Korea

Tel: +82-51-240-7675, Fax: +82-51-246-8668

E-mail: voiceleebj@gmail.com
}

quate visual field resulting from hemorrhage, anatomic variations, or insufficient surgeon experience. Such nerve injuries can reduce the quality of life of patients and cause legal problems. In thyroid surgery, unilateral vocal fold palsy (VFP) may cause hoarseness, aspiration, and other adverse events that could reduce the quality of life. In the case of bilateral VFP palsy, a tracheostomy may be necessary. It has been reported that the incidence of nerve injury varies from $2.3 \%$ to $26 \%$ in thyroid surgery [1]. Another study reported that the rate of transient and consistent VFP after thyroid surgery was $5.2 \%$ and $1.4 \%$, respectively [2]. Most cases of recurrent laryngeal nerve (RLN) damage are caused by traction, compression, thermal injury,

Copyright (C) 2019 by Korean Society of Otorhinolaryngology-Head and Neck Surgery.

This is an open-access article distributed under the terms of the Creative Commons Attribution Non-Commercial License (http://creativecommons.org/licenses/by-nc/4.0)

which permits unrestricted non-commercial use, distribution, and reproduction in any medium, provided the original work is properly cited. 
clamping, ischemia, or transection $[3,4]$.

An objective method to prevent nerve injury is needed to identify the nerves and determine their status during surgery. Intraoperative neuromonitoring (IONM) has been used to reduce the risk of nerve damage during spine, thyroid, otologic, or parotid surgery. IONM is performed in thyroidectomy to identify RLN early, map the nerve pathways according to anatomical variations, reduce mechanical trauma to the nerve, and assess the function and prognosis of the RLN [3,5,6]. In recent years, about $40 \%-45 \%$ of the surgeons are using IONM for thyroid or parathyroid surgery [7].

IONM, which is used in thyroid surgery, is a method of confirming the state of the nerve by measuring the electromyography (EMG) signals of the muscle in which the nerve is distributed. The identification of RLN using EMG of the vocalis muscles during thyroidectomy may be helpful. There are recent reports about the usefulness of IONM in thyroid surgery for reducing the rate of temporary VFP [8-10]. However, even with the use of IONM in thyroid surgery, VFP might still occur. The use of IONM did not result in a statistically significant reduction in the incidence of permanent VFP. Consequently, the use of IONM during thyroid surgery remains controversial $[8,11]$.

Dralle et al. [11], in their systematic review about the utility of thyroid IONM, reported high negative predictive values (NPVs; $92 \%-100 \%$ ) but relatively low and variable positive predictive values (PPVs; 10\%-90\%). The widely variable PPV is one of the reasons for the controversy about the usefulness and accuracy of IONM during thyroid surgery. In IONM using endotracheal tube (ETT)-based electrodes, proper contact between the surface electrodes of the ETT and the vocal cords is crucial $[12,13]$. The contact may be affected by the patient's postural changes, thyroid nodule size, and excessive pooling of saliva or sputum [14]. If the contact between the ETT electrodes and the vocal cords is not precise owing to positional changes of the patient or the rotation of the ETT, the EMG signals of the vocalis muscle cannot be measured even if the status of the RLN is normal. Although loss of signal (LOS), which does not show the EMG responses of the vocalis muscle, may be observed when stimulating the RLN during surgery, the movement of the vocal cords may still be normal after surgery. Thus, if LOS

\section{H I G H L I G H T S}

- The risk of nerve damage may occur due to false-negative or false-positive responses on conventional intraoperative neuromonitoring (IONM).

- We developed a novel IONM system using a surface pressure sensor which is to measure the change of surface pressure using a piezo-electric effect instead of electromyography to detect muscle twitching.

- The application of IONM using a surface pressure sensor during surgery is noninvasive, safe, and feasible. occurs in the IONM system during thyroid surgery, laryngeal muscle twitching should be assessed by surgeons with their fingers to confirm the accuracy of EMG. Furthermore, instead of using EMG recordings to identify the location and state of the RLN or facial nerve, it is possible to investigate muscle twitching on the mucosa or skin.

Our novel device uses piezo-electric sensor to measure muscle twitching while reducing false positive or false negative of EMG. Piezo-electric effect converts changes in pressure, acceleration, temperature, strain, or force into an electrical charge. Piezoelectric sensors are widely used in medical and industrial applications such as middle ear implants, ultrasonic transducers, and electric musical instruments. The bio-pressure sensor using piezo-electric effect measure changes in pressure by converting these changes into an electrical charge. Piezoelectric sensors are used in everyday life and are safe.

Therefore, measuring muscle twitching by using the novel IONM system, which does not require the surgeon to directly touch the muscle to identify the state of nerves, can be an alternative to recording of EMG responses. The present study aimed to investigate the feasibility and safety of the developed IONM system using a patch-type piezo-electric sensor to detect the changes in the surface pressure or vibration of the skin due to muscle twitching induced by nerve stimulation in a rabbit model.

\section{MATERIALS AND METHODS}

\section{Animals}

The experimental protocol was approved by the Pusan National University Institutional Animal Care and Use Committee (No. PNUYH-2018-51). The experiment was performed on six sciatic nerves in three New Zealand white female rabbits (3-4 kg). After overnight fasting, the rabbits were sedated with an intramuscular injection of ketamine hydrochloride $(10 \mathrm{mg} / \mathrm{kg})$ and xylazine $(2 \mathrm{mg} / \mathrm{kg})$, and anesthesia was maintained with inhaled 3\% isoflurane. After the rabbits were anesthetized, they were placed in a prone position while voluntary respiration was maintained. Both femoral regions were shaved and disinfected with betadine solution, followed by local anesthesia with $1 \%$ lidocaine and skin incision. The sciatic nerve was exposed.

\section{Study design}

The largest amplitudes of the EMG and piezo-electric surface pressure sensor were captured and registered at every step; the latencies of the EMG and piezo-electric surface pressure sensor were recorded at the largest amplitudes. All rabbits were monitored using a Nerve Integrity Monitor (NIM-Response 3.0 System; Medtronic Xomed, Jacksonville, FL, USA) and a handmade surface pressure sensor monitor. A pair of EMG needle electrodes were inserted into a gastrocnemius muscle to check the EMG signals. Simultaneously, a patch electrode of the sur- 

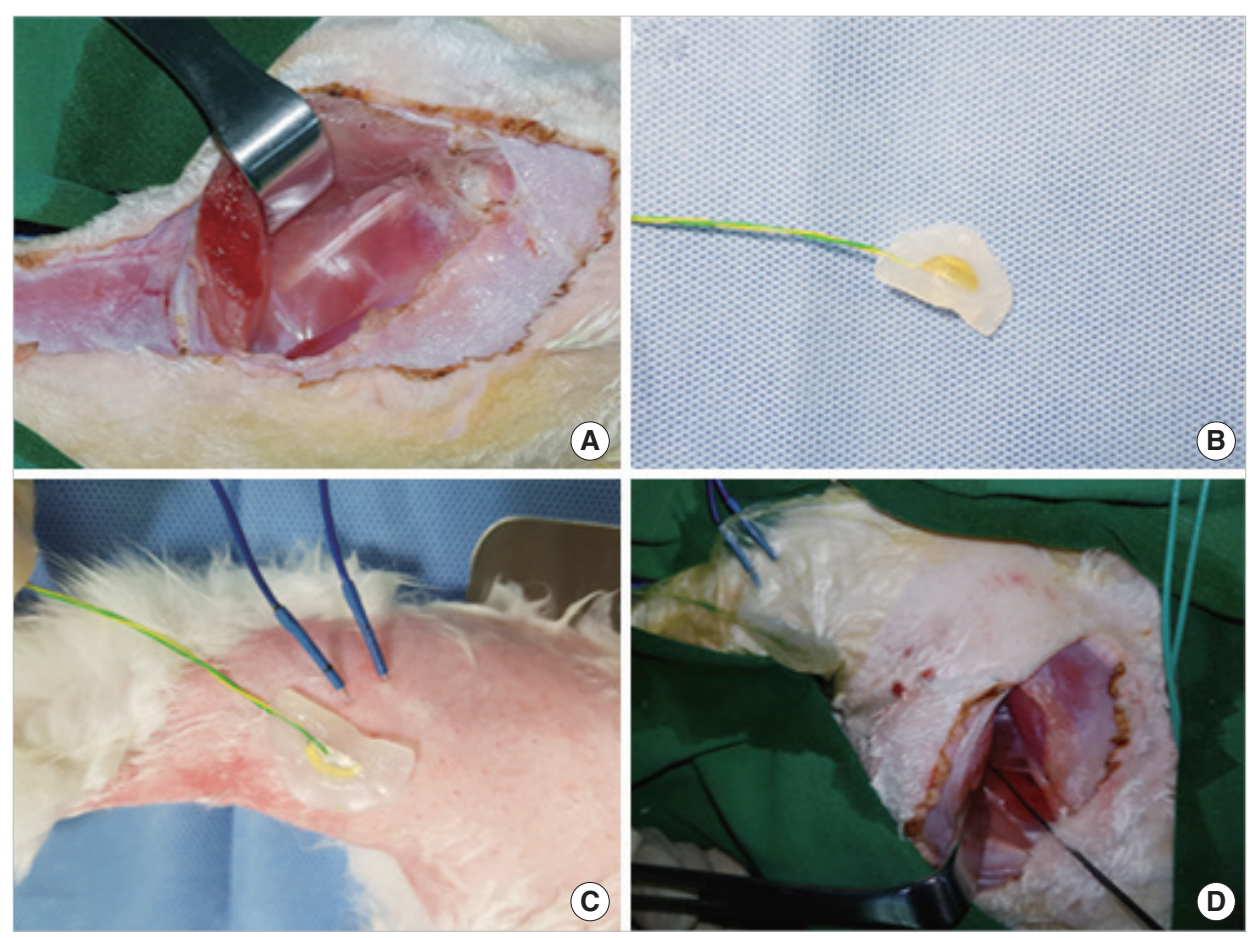

Fig. 1. Photographs of the surgical field on the rabbit, demonstrating dissection using the surface pressure sensor. (A) Left sciatic nerve. (B) Modified patch-type piezo-electric surface pressure sensor. (C) A needle-electrode electromyography and modified patch-type piezo-electric surface pressure sensor on the skin. (D) Sciatic nerve stimulation with the nerve probe.

Table 1. Analysis of the amplitude and latency of the EMG and surface pressure sensor depending on the value of the sciatic nerve stimulus in rabbits

\begin{tabular}{|c|c|c|c|c|c|c|c|c|}
\hline \multirow{2}{*}{ Stimulus (mA) } & \multicolumn{3}{|c|}{ EMG } & \multicolumn{3}{|c|}{ Surface pressure sensor } & \multicolumn{2}{|c|}{$P$-value ${ }^{a)}$} \\
\hline & Response (n) & Amplitude $(\mu \mathrm{V})$ & Latency (ms) & Response (n) & Amplitude $(\mu \mathrm{V})$ & Latency (ms) & Amplitude & Latency \\
\hline 0.02 & 1 & 681 & 3.5 & 1 & 144 & 26 & - & - \\
\hline 0.03 & 1 & 337 & 7.7 & 0 & - & - & - & - \\
\hline 0.05 & 5 & $812.4 \pm 840.7$ & $5.4 \pm 1.8$ & 5 & $312.2 \pm 351.0$ & $34.6 \pm 3.7$ & $<0.001$ & $<0.001$ \\
\hline 0.1 & 6 & $2,567.8 \pm 3,806.1$ & $5.7 \pm 1.6$ & 6 & $525.6 \pm 519.3$ & $30.0 \pm 4.3$ & $<0.001$ & $<0.001$ \\
\hline 0.5 & 6 & $3,957.0 \pm 4,511.0$ & $6.2 \pm 1.9$ & 6 & $532.0 \pm 346.8$ & $26.4 \pm 3.7$ & $<0.001$ & $<0.001$ \\
\hline 1.0 & 6 & $3,978.3 \pm 2,977.3$ & $5.9 \pm 1.8$ & 6 & $686.3 \pm 369.4$ & $26.4 \pm 2.5$ & $<0.001$ & $<0.001$ \\
\hline 2.0 & 6 & $2,608.6 \pm 464.7$ & $6.5 \pm 2.2$ & 6 & $751.6 \pm 448.0$ & $27.7 \pm 2.5$ & $<0.001$ & $<0.001$ \\
\hline 3.0 & 6 & $2,088.4 \pm 1,338.8$ & $6.5 \pm 2.2$ & 6 & $785.2 \pm 413.5$ & $27.1 \pm 2.5$ & $<0.001$ & $<0.001$ \\
\hline Total & & $2,598.6 \pm 2,850.9$ & $6.0 \pm 1.8$ & & $584.0 \pm 413.5$ & $28.1 \pm 3.6$ & $<0.001$ & $<0.001$ \\
\hline
\end{tabular}

Values are presented as mean \pm standard deviation.

EMG, electromyography.

a) $P$-value indicates the result of the comparison test for the mean response values of the amplitude and latency between the EMG and surface pressure sensor depending on stimulus value.

face pressure sensor was attached to the skin above the gastrocnemius muscle (Fig. 1). After the completion of the experiment, the anesthetized rabbits were euthanized with intravenous Potassium chloride injection.

We developed a novel hand-made IONM system using a surface pressure sensor for motor nerve preservation. The sciatic nerve was stimulated once with the nerve stimulator for each stimulus value, by using the NIM 3.0 system. The stimulation threshold was displayed as the stimulus value at which the re- sponse first appears. The average amplitudes of the EMG and surface pressure sensor on nerve stimulation by the nerve probe were compared. The experiments were performed to investigate the safety and feasibility of use of the surface pressure sensor for IONM. We evaluated the amplitude and latency of the EMG and surface pressure sensor recordings by using a nerve probe in six sciatic nerves of three rabbits (Table 1 ). The mean values and standard deviations were then calculated. 

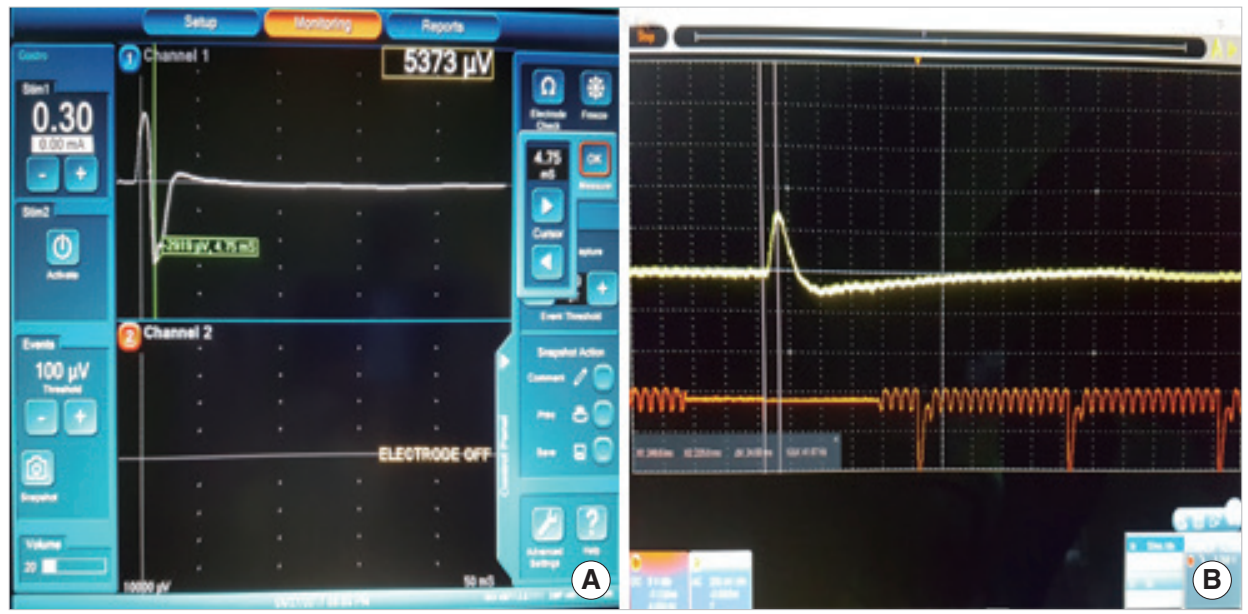

Fig. 2. Electromyography (EMG) and piezo-electric surface pressure sensor recordings, showing the amplitude and latency of the EMG and surface pressure sensor for $0.3 \mathrm{~mA}$ stimulation using the Nerve Integrity Monitor (NIM) 3.0 system in one rabbit. (A) EMG signal. (B) The modified piezo-electric surface pressure sensor signal.

\section{Surface pressure sensor}

This device employs a piezo-electric effect. Piezo-electric sensors measure changes in pressure, acceleration, temperature, strain, or force by converting these changes into an electrical charge. The novel device was modified to measure the change in pressure by using a piezo-electric button sensor to detect muscle movement (Fig. 1B). The change in the skin surface pressure caused by muscle movement was measured with a skin-mounted surface pressure sensor.

Because this is a patch-type device, it attaches to the skin without inserting a needle electrode into the muscle. On application, surface pressure is generated by the movement of the corresponding muscle in response to nerve stimulation. Consequently, the modified piezo-electric surface pressure sensor can measure the signal amplitude and latency of the muscle twitching.

\section{Statistical analysis}

Statistical analyses were performed using the nonparametric Mann-Whitney $U$-test in IBM SPSS ver. 21.0 (IBM Corp., Armonk, NY, USA). A $P$-value of $<0.05$ was considered statistically significant.

\section{RESULTS}

The amplitude and latency recordings of the EMG and surface pressure sensor from six sciatic nerves were analyzed (Table 1 , Fig. 2). The use of the surface pressure sensor was feasible and safe in all cases. At $0.02 \mathrm{~mA}$ stimulation, the amplitude of the EMG and surface pressure sensor signals was $681 \mu \mathrm{V}$ and $144 \mu \mathrm{V}$, respectively, in one sciatic nerve, and the latency was $3.5 \mathrm{~ms}$ and $26 \mathrm{~ms}$, respectively. For $0.03 \mathrm{~mA}$ stimulation, an EMG amplitude of $337 \mu \mathrm{V}$ and a latency of $7.7 \mathrm{~ms}$ were observed for an- other sciatic nerve. EMG and surface pressure sensor responses to $0.05 \mathrm{~mA}$ stimulation in five of six sciatic nerves were noted. The surface pressure sensor detected muscle movement in response to $0.1 \mathrm{~mA}$ stimulation in all six sciatic nerves. These stimuli are very faint compared with those used in parotidectomy or thyroidectomy. As Table 1 shows, there was no difference in the thresholds for stimulus intensity between the novel device and $\mathrm{EMG}$ in the detection of muscle movement.

The average amplitudes of the EMG and surface pressure sensor for the sciatic nerve were 2,598.6 $\pm 2,850.9 \mu \mathrm{V}$ and $584.0 \pm$ $413.5 \mu \mathrm{V}$, respectively. The average latencies of the EMG and surface pressure sensor for the sciatic nerve were $6.0 \pm 1.8 \mathrm{~ms}$ and $28.1 \pm 3.6 \mathrm{~ms}$, respectively. The mean amplitude difference of EMG and the surface pressure sensor was $2,000 \mu \mathrm{V}$, and the mean latency time difference was $22 \mathrm{~ms}$. As shown in Table 1, there were significant differences in the signal amplitude and latency between EMG and the surface pressure sensor depending on the stimulus value $(P<0.001$ and $P<0.001$, respectively). A direct comparison of the difference in amplitude is meaningless because of the difference in the measurement methods. There was an average difference of $22 \mathrm{~ms}$ in the latency; however, during parotid or thyroid surgery, a delay in the response by 0.022 seconds is clinically trivial.

\section{DISCUSSION}

RLN injury is the most important complication in thyroid surgery. The best way to prevent damage to the RLN is to identify its location and assess its status during surgery. Thus, IONM was developed using ETT-based surface electrodes to easily identify the status of the RLN. IONM using ETT-based surface electrodes was believed to reduce RLN damage. However, a recent meta- 
analysis reported that the effect of IONM on reducing the incidence of temporary and permanent VFP was not statistically significant [15]. In addition, although IONM has a very high NPV (92\%-100\%), its PPV is highly variable (10\%-90\%) $[16,17]$. Thus, nerve injury cannot be completely predicted even with LOS from IONM during thyroid surgery.

To preserve the nerves during surgery, IONM is used to identify the nerves by measuring their function, through recording of evoked potentials, electroencephalography, or EMG, which apply electrical stimulation to structures suspected to be nerves. The IONM system comprises an electric stimulator, which is a nerve probe; a response detector, such as evoked potentials, electroencephalography, or EMG; and a neural monitoring system, including a monitor and a pulse generator. IONM of the thyroid is a method of evaluating muscle movement by using EMG upon the application of electric stimulation to the nerve. In general, there are two types of electrodes for EMG measurement: one is a needle electrode inserted into the muscle used for EMG, such as that used during parotid surgery, and the other is an electrode attached to the muscle surface to measure the activity potential through a surface derivation method, such as that used during thyroid surgery.

There are some limitations on stimulating side and recording side during thyroid surgery under IONM. To overcome the problems of the stimulation side, the continuous IONM system was introduced [6], and attempts were made to connect the nerve stimulator with the surgical instrument [18-20]. But, there were few attempts to overcome the disadvantages of recording side like EMG during thyroid surgery.

It is not easy to measure EMG signals with the ETT-based surface electrodes used for thyroid surgery because these electrodes are very sensitive to changes in contact with the muscle and to the pooling of saliva or sputum around them [14]. EMG evaluates the movement of muscles by measuring the potential difference between at least two electrodes. If either of the two electrodes is disconnected from contact with the muscle because of positional changes of the patient or because of the rotation of the ETT, the EMG signals cannot be measured or the measurement becomes inaccurate [5]. Moreover, it is troublesome to check whether the electrode is precisely attached to the vocal cords by using a laryngeal fiberscope after anesthesia [12,21]. EMG signals can also be missed or misinterpreted during monitoring owing to the depth of anesthetic effect, neuromuscular blockers, electrocauterization, or cold-water stimulation, as well as owing to errors in the EMG monitoring setup [22-26]. Even if LOS is observed during surgery, there is no VFP after surgery in some cases [5,27]. Hence, the use of IONM during thyroidectomy for predicting and preventing intraoperative and postoperative nerve injury remains controversial.

The risk of nerve damage may be associated with false-negative or false-positive responses in IONM during thyroid surgery. Disagreements between intraoperative EMG findings and post- operative RLN function can be explained by several factors. Among them are equipment problems that can occur during stimulation and the recording side. False positives should be minimized because these can cause excessive anxiety to the surgeon; similarly, false negatives should also be minimized as these could make the surgeon excessively bold. Furthermore, IONM may provide false information suggesting that the nerve is safe, which could lead to a less meticulous dissection of the nerve during thyroidectomy. Therefore, the surgeon should not rely on EMG signals alone and must analyze the operation field with an anatomical understanding. If an abnormal signal is observed in the IONM system, laryngeal twitching should be checked using the surgeon's finger to confirm the accuracy of the EMG signals.

A new IONM system for measurement of muscle movements that is less affected by positional changes or electrode contact with nerve stimulation is needed. Therefore, we developed a novel IONM system using a patch-type piezo-electric surface pressure sensor that measures the changes in pressure, acceleration, temperature, strain, or force by converting these changes into an electrical charge. The piezo-electric sensor was modified to measure the pressure change in the skin or mucosal surface to detect muscle twitching. The objective of this study was to investigate the feasibility and safety of the novel IONM system using a surface pressure sensor in an experimental animal model before its application to human patients. We analyzed the detection thresholds, as well as the amplitude and latency of the surface pressure sensor and EMG signals of the sciatic nerve to evaluate the feasibility of the novel IONM system. The novel device and EMG responded to a $0.1 \mathrm{~mA}$ stimulus in all cases, indicating no differences in stimulus thresholds between the novel device and EMG in detecting muscle movement. The amplitude of the surface pressure sensor is smaller than that of the EMG; nonetheless, the use of this device is feasible, as shown in Table 1, because there are no differences in the detection thresholds for muscle movement. The mean latency time of the surface pressure sensor was $22 \mathrm{~ms}$ slower than that of the EMG, which means that the mean response time of the EMG is $0.022 \mathrm{sec}$ onds faster than that of the surface pressure sensor; however, this difference is not clinically meaningful.

This novel device is expected to overcome some of the problems that occur during surgery with IONM using EMG. EMG evaluates muscle movement by measuring the action potential difference between two electrodes. Thus, if the electrode moves to a different site or the contact of the electrode with the muscle changes (owing to positional changes of the patient or rotation of the ETT), muscle movement cannot be accurately evaluated with EMG. However, a patch-type device can assess muscle movement or twitching by being in contact with only a small part of the surface pressure sensor on the ETT or skin surface, if the patch type bio-pressure sensor is wound around the ETT. The novel IONM with a patch-type piezoelectric surface pressure sensor can also prevent complications related to electrode 
needle insertion, such as bleeding, infection, or pain. In addition, because this device may be responsive to the pressure change in the skin or mucosal surface that appears in response to stimulation, more accurate nerve monitoring can be achieved while reducing the rate of false positives and false negatives caused by electrocauterization or cold irritation.

This study has some limitations. First, the amplitude and latency time of the novel device are significantly different from those of EMG. However, the difference in amplitude is not meaningful because of the difference in the measurement methods. Furthermore, a 0.022-second delay in response is clinically meaningless during thyroid or parotid surgery. From the perspective of nerve preservation, the piezo-electric surface pressure sensor does not differ from EMG in that it responds accurately to small stimuli. Second, as this study is an animal study, a human study needs to be conducted to verify the validity and safety of this method.

It is possible to measure the surface pressure changes by using a piezo-electric surface pressure sensor instead of EMG to evaluate muscle movements induced by nerve stimulation. The novel IONM system with a piezo-electric surface pressure sensor can be an alternative to EMG-based IONM.

\section{CONFLICT OF INTEREST}

No potential conflict of interest relevant to this article was reported.

\section{ACKNOWLEDGMENTS}

This work was supported by the Korean Thyroid Association Outstanding Investigator Award 2018, Seoul, Republic of Korea. The award organization had no role in study design and conduct, collection, analysis, and interpretation of the data, and writing or approval of the manuscript.

\section{ORCID}

Eui-Suk Sung https://orcid.org/0000-0001-8752-3426

Byung-Joo Lee https://orcid.org/0000-0001-7091-6688

\section{REFERENCES}

1. Jeannon JP, Orabi AA, Bruch GA, Abdalsalam HA, Simo R. Diagnosis of recurrent laryngeal nerve palsy after thyroidectomy: a systematic review. Int J Clin Pract. 2009 Apr;63(4):624-9.

2. Lo CY, Kwok KF, Yuen PW.A prospective evaluation of recurrent laryngeal nerve paralysis during thyroidectomy. Arch Surg. 2000 Feb; 135(2):204-7.
3. Chiang FY, Lu IC, KuoWR, Lee KW, Chang NC, Wu CW. The mechanism of recurrent laryngeal nerve injury during thyroid surgery: the application of intraoperative neuromonitoring. Surgery. 2008 Jun; 143(6):743-9.

4. Dionigi G, Alesina PF, Barczynski M, Boni L, Chiang FY, Kim HY, et al. Recurrent laryngeal nerve injury in video-assisted thyroidectomy: lessons learned from neuromonitoring. Surg Endosc. 2012 Sep;26(9): 2601-8.

5. Randolph GW, Dralle H; International Intraoperative Monitoring Study Group, Abdullah H, Barczynski M, Bellantone R, et al. Electrophysiologic recurrent laryngeal nerve monitoring during thyroid and parathyroid surgery: international standards guideline statement. Laryngoscope. 2011 Jan;121 Suppl 1:S1-16.

6. Chiang FY, Lee KW, Chen HC, Chen HY, Lu IC, KuoWR, et al. Standardization of intraoperative neuromonitoring of recurrent laryngeal nerve in thyroid operation. World J Surg. 2010 Feb;34(2):223-9.

7. Horne SK, Gal TJ, Brennan JA. Prevalence and patterns of intraoperative nerve monitoring for thyroidectomy. Otolaryngol Head Neck Surg. 2007 Jun;136(6):952-6.

8. Barczynski M, Konturek A, Cichon S. Randomized clinical trial of visualization versus neuromonitoring of recurrent laryngeal nerves during thyroidectomy. Br J Surg. 2009 Mar;96(3):240-6.

9. Dralle H, Sekulla C, Haerting J,TimmermannW, Neumann HJ, Kruse E, et al. Risk factors of paralysis and functional outcome after recurrent laryngeal nerve monitoring in thyroid surgery. Surgery. 2004 Dec;136(6):1310-22.

10. Angelos P. Ethical and medicolegal issues in neuromonitoring during thyroid and parathyroid surgery: a review of the recent literature. Curr Opin Oncol. 2012 Jan;24(1):16-21.

11. Dralle H, Sekulla C, Lorenz K, Brauckhoff M, Machens A; German IONM Study Group. Intraoperative monitoring of the recurrent laryngeal nerve in thyroid surgery. World J Surg. 2008 Jul;32(7):135866.

12. Tsai CJ,Tseng KY,Wang FY, Lu IC,Wang HM,Wu CW, et al. Electromyographic endotracheal tube placement during thyroid surgery in neuromonitoring of recurrent laryngeal nerve. Kaohsiung J Med Sci. 2011 Mar;27(3):96-101.

13. Kim HY,Tufano RP, Randolph G, Barczynski M,Wu CW, Chiang FY, et al. Impact of positional changes in neural monitoring endotracheal tube on amplitude and latency of electromyographic response in monitored thyroid surgery: results from the porcine experiment. Head Neck. 2016 Apr;38 Suppl 1:E1004-8.

14. Chiang FY, Lu IC, Chang PY, Dionigi G, Randolph GW, Sun H, et al. Comparison of EMG signals recorded by surface electrodes on endotracheal tube and thyroid cartilage during monitored thyroidectomy. Kaohsiung J Med Sci. 2017 Oct;33(10):503-9.

15. Higgins TS, Gupta R, Ketcham AS, Sataloff RT, Wadsworth JT, Sinacori JT. Recurrent laryngeal nerve monitoring versus identification alone on post-thyroidectomy true vocal fold palsy: a meta-analysis. Laryngoscope. 2011 May;121(5):1009-17.

16. Barczynski M, Konturek A, Pragacz K, Papier A, Stopa M, NowakW. Intraoperative nerve monitoring can reduce prevalence of recurrent laryngeal nerve injury in thyroid reoperations: results of a retrospective cohort study.World J Surg. 2014 Mar;38(3):599-606.

17. Yang S, Zhou L, Lu Z, Ma B, Ji Q, Wang Y. Systematic review with meta-analysis of intraoperative neuromonitoring during thyroidectomy. Int J Surg. 2017 Mar;39:104-13.

18. Sung ES, Lee JC, Shin SC, Choi SW, Jung DW, Lee BJ. Development of a novel detachable magnetic nerve stimulator for intraoperative neuromonitoring. World J Surg. 2018 Jan;42(1):137-42.

19. Sung ES, Lee JC, Kim SH, Shin SC, Jung DW, Lee BJ. Development of an attachable endoscopic nerve stimulator for intraoperative neuromonitoring during endoscopic or robotic thyroidectomy. Otolaryngol Head Neck Surg. 2018 Mar;158(3):465-8. 
20. Shin SC, Sung ES, Choi SW, Kim SD, Jung DW, Kim SH, et al. Feasibility and safety of nerve stimulator attachment to energy-based devices: a porcine model study. Int J Surg. 2017 Dec;48:155-9.

21. Lu IC, Chu KS, Tsai CJ, Wu CW, Kuo WR, Chen HY, et al. Optimal depth of NIM EMG endotracheal tube for intraoperative neuromonitoring of the recurrent laryngeal nerve during thyroidectomy. World J Surg. 2008 Sep;32(9):1935-9.

22. Meier JD, Wenig BL, Manders EC, Nenonene EK. Continuous intraoperative facial nerve monitoring in predicting postoperative injury during parotidectomy. Laryngoscope. 2006 Sep;116(9):1569-72.

23. Dulguerov P, Marchal F, Lehmann W. Postparotidectomy facial nerve paralysis: possible etiologic factors and results with routine facial nerve monitoring. Laryngoscope. 1999 May;109(5):754-62.
24. Grosheva M, Guntinas-Lichius O. Significance of electromyography to predict and evaluate facial function outcome after acute peripheral facial palsy. Eur Arch Otorhinolaryngol. 2007 Dec;264(12): 1491-5.

25. Anon JB, Lipman SP, Guelcher RT, Sibly DA, Thumfart W. Monitoring the facial nerve during parotidectomy. Arch Otolaryngol Head Neck Surg. 1991 Dec;117(12):1420.

26. Empis de Vendin O, Schmartz D, Brunaud L, Fuchs-Buder T. Recurrent laryngeal nerve monitoring and rocuronium: a selective sugammadex reversal protocol.World J Surg. 2017 Sep;41(9):2298-303.

27. Wu CW, Wang MH, Chen CC, Chen HC, Chen HY, Yu JY, et al. Loss of signal in recurrent nerve neuromonitoring: causes and management. Gland Surg. 2015 Feb;4(1):19-26. 